\title{
Efficient Price and Utility based Congestion Control in the Next Generation Networks 4G
}

\author{
Kafilu Barau Danbatta \\ Nigerian Communications Commission \\ Plot 426 Aguiyi Ironsi Street Maitama Abuja
}

\author{
Umar Garba Danbatta \\ Department of Electrical Engineering \\ Bayero University Kano
}

\begin{abstract}
In the Next Generation Networks like 4G, it is highly essential to create a market mechanism that would allow the customer to communicate with network and negotiate a contract based on some QoS parameters like blocking probability, delay, arrival rate, spectral efficiency, resource allocation and price. However, the mechanisms, rather than technical-oriented scheme, that involve the use of economic theories may provide better solutions to accommodate the high demand of mobile services. The purpose of this research work is to propose and validate mathematical models that study the effect of pricing incentives as an additional strategy for encouraging a more efficient usage of limited network resources. This paper has examined a real-time resource trading between the customers and network providers based on principles of economic utility, pricing and QOS. A price and utility-based CAC scheme using combined concepts from network, engineering design, concepts from economics and user behavior has been mathematically formulated to provide an overall call admission strategy that simultaneously alleviates the network congestion, meets the QoS requirements of users, increases the network operator revenue and uses the network resources efficiently. The parameterized utility and acceptance probability functions that explicitly represent the interactions and negotiations of resource allocations between a Next Generation Network and its wireless users have been developed and validated. The performance of the proposed integrated approach has been compared with the existing model. The Cumulative Resource Efficiency (CRE) index in the proposed pricing and utility scheme has outperformed the existing model by $6 \%$.
\end{abstract}

General Terms: Price and Utility based Congestion Control, Multi-Criteria-Multi-Quadrant (MCMQ) approach, Total Revenue Generated, Total Allocated Resources, and Cumulative Resource Allocated (CRA).

Keywords: Cumulative Resource Efficiency Index (CRI), Cumulative Revenue (CR), Acceptance Probability, Utility, Low Priority User (LPU) and High Priority User (HPU).

\section{INTRODUCTION}

The incentives used to influence the demand of network services involve how the network operators set up pricing schemes. Most pricing schemes are based on the supplydemand relationship analysis in economics. Generally, user's demand will decrease with the increase of price while supply will increase and vice-versa. Each network user expects certain demands from the networks. The level of the user's demand is identified by a utility function which describes a level of user satisfaction as a function of network resource. The network operators need to offer prices to network users appropriately so that they can generate high revenue and meet the expectation of the users. To achieve such goals, price for network services must change in response to the fluctuation of demand. The relationship between demand and prices needs to be identified in order to give the right signal for network users to adjust their usage. This relationship is best identified by a demand function, i.e. the reaction of users to the change of price. This implies whether users are either encouraged or discouraged to use the network as price changes [1]. Various demand functions have been proposed in the literature. Most of the existing literatures have utilized the demand function, based on the research work of [2], who have investigated user behavior with regard to differential pricing. The demand function is used for different user priority classes in separate networks. This paper proposed an efficient demand function which has been proved to outperform the existing ones.

The emergence of mobile technologies as a new platform for computation and communication has contributed to the development and deployment of new mobile services. Creating a market mechanism that would allow the customer to communicate with Network and negotiate a contract based on some QoS parameters like blocking probability, delay, arrival rate, spectral efficiency, rate allocation, price, etc. Pricing can be used for signaling the congestion levels in different wireless networks and to let users communicate their willingness-to-pay to the market [3].

\section{TWO UTILITY AND ACCEPTANCE PROBABILITY MODELS FOR UNIVERSAL APPLICATIONS}

In this section two Utility and Acceptance Probability functions for Universal Application are introduced:

1) Vuong Q.N (2008) utilized this Utility function in the Network Selection Criteria.

$$
U(x)=\left\{\begin{array}{cc}
\frac{x-x_{\min }}{\left(\frac{x-x_{\min }}{x_{m}}\right)}, & x<x_{\min } \leq x \leq x_{\max } \\
1+\left(\frac{x-x_{\min }}{x_{m}-x_{\min }}\right)^{\delta} & \\
1-\frac{\left(\frac{x_{\max }-x}{x_{\max }-x_{m}}\right)^{\gamma}}{1+\left(\frac{x_{\max }-x}{x_{\max }-x_{m}}\right)^{\gamma}}, & x_{m} \leq x \leq x_{\max } \\
1 & x>x_{\max }
\end{array}\right.
$$

2) The original Cobb-Douglas acceptance probability has the following form:

$$
A_{j}^{v b r}\left(b_{j}^{v b r}, p_{j}^{v b r}\right)=1-e^{-K\left[U_{b j}{ }^{v b r}(b j)^{\mu}\right]}\left(\frac{p_{j}^{v b r}}{\varnothing}\right)^{-\epsilon}
$$




$$
A_{i}^{c b r}\left(b_{i}^{c b r}, p_{i}^{c b r}\right)=1-e^{-K\left[U_{b i}{ }^{c b r}(b i)^{\mu}\right]}\left(\frac{p_{i}^{c b r}}{\varnothing}\right)^{-\epsilon}
$$

This is widely used in different disciplines like economics, operation research, etc. These two utility functions shall be used for our performance and comparative analysis.

The revenue $\mathrm{R}$ earned by the provider, the number $\mathrm{S}$ of admitted users, the amount $\mathrm{T}$ of allocated resource and the total utility $\mathrm{U}$ for admitted users can be computed respectively [5] as :

$$
\begin{aligned}
R & =\sum_{i=1}^{N} p_{i} A\left(u_{i}, p_{i}\right) \\
S & =\sum_{i=1}^{N} A\left(u_{i}, p_{i}\right) \\
T & =\sum_{i=1}^{N} r_{i} A\left(u_{i}, p_{i}\right) \\
U & =\sum_{i=1}^{N} u_{i} A\left(u_{i}, p_{i}\right)
\end{aligned}
$$

where $p_{i}$ s are the flat prices paid by all users in the same class of service. In this formula, $A\left(u_{i}, p_{i}\right)$ is the acceptance probability of user $i$ and it has been defined as a tradeoff between the perceived QoS and the price to be paid [7]. It is an increasing function of the utility and a decreasing function of the price. This is generally computed on bandwidth and price and is widely used in the computation of the operator's payoff in radio resource management.

\section{PRICE AND UTILITY BASED}

\section{CONGESTION CONTROL ALGORITHM}

Since in the conventional schemes, all users accept a quoted price by a Network Service Provider (NSP), no fairness is achieved despite the fact that it would sometimes generate higher revenues. In this scheme, we assume that the blocking probability is constant and the resources are reasonably available. The Network is not in a congestion state. The users would tend to be more satisfied and this would prevent the network from getting into congestion state easily as the price ranges offered by the NSP would induce a positive or negative incentive to the utilization of the scarce resources. The range of the prices quoted by the NSP should be reviewed by the Nigerian Communications Commission NCC as a regulator.

When evaluating the utility of an access network, upward $U^{u}(x)$ and downward $U^{d}(x)$ criteria should be distinguished. A criterion is classified as upward if its utility is an increasing function of its value. Upward criteria include parameters such as allocated bandwidth, throughput, reliability degree, and Receive Signal Strength (RSS). Conversely, the utility of a downward criterion decreases in function of its value. Downward criteria include parameters such as network usage cost, energy consumption, bit error rate, transfer delay and handover frequency. Price is not only the network usage cost but also, for example, the power dissipation at the terminal side. [7].

The acceptance probability based on price criterion is proposed as;

$$
\begin{aligned}
& A\left(\boldsymbol{p}_{i}^{\text {traffic class }}(t)\right)= \\
& \left(1-\left(\frac{p_{i}^{\text {traff class }}(t)-p_{\min }^{\text {traff class }}}{p_{\text {max }}^{\text {trafic }}{ }^{\text {trass }}-p_{\min }^{\text {traffic class }}}\right)^{\alpha}\right)
\end{aligned}
$$

Then, using the relationship (Upward Criterion $=1-$ Downward Criterion), the acceptance probability of the band width criterion which is upward could be computed as;

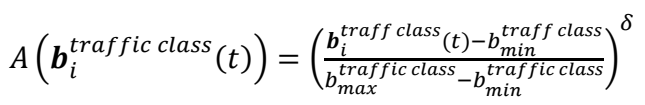

Equations (8) and (9) represent the acceptance probabilities in the two quadrants, only. For the four quadrants, some additional parameters are introduced as follows; Centers of Utility; $g^{q 1}, g^{q 2}, g^{q 3}, g^{q 4}$; price at the centers of utility; $p_{m}$, bandwidth at the centers of utility; $b_{m}$ and acceptance probabilities at the intervals $p<p_{\min } ; \quad p_{\min } \leq p \leq p_{m}$ $p_{m} \leq p \leq p_{\max } ; p>p_{\max } ; b<b_{\min } ; \quad b_{\min } \leq b \leq b_{m}$; $b_{m} \leq b \leq b_{\max } ; b>b_{\max }$. This can be explained explicitly from table1.The center of utility equals 0.5 has been chosen, however other values like 0.6 or 0.4 could be chosen depending on the service application of a traffic class. Then after some little modifications based on the above arguments and the two quadrant approach, the utilities for upward criterion $U^{u}(x)$ and downward criterion $U^{d}(x)$ in the four quadrants are proposed as follows;

$$
\begin{aligned}
& U^{d}(x)
\end{aligned}
$$

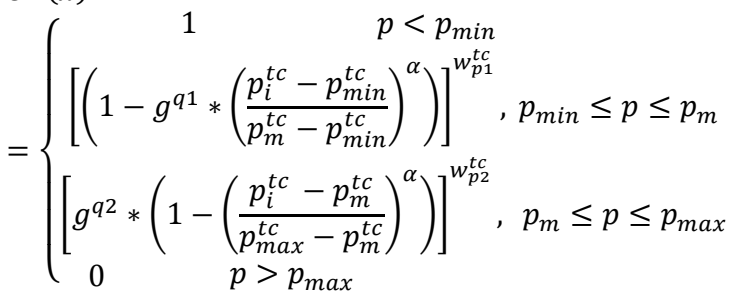

Where,

$p_{i}^{t c}$ is the price paid by admitted traffic class in the first quadrant; $p_{\text {max }}^{t c}$ is the maximum price quoted to a traffic class in the first quadrant; $p_{\text {min }}^{t c}$ is the minimum price quoted to a traffic class in the first quadrant; $w_{p 1}^{t c}$ is the preference weight for a price of a traffic class in the first quadrant; $w_{p 2}^{t c}$ is the preference weight for a price of a traffic class in the second quadrant; $g^{q 1}=g^{q 2}=0.5$ are the centers of the utility, $\alpha$ is the user price sensitivity index.

$$
\begin{aligned}
& U^{u}(x) \\
& {\left[g^{q 3} *\left(\frac{b_{j}^{t c}-b_{\text {min }}^{t c}}{b_{m}^{t c}-b_{\text {min }}^{t c}}\right)^{\delta}\right]^{w_{b 3}^{t c}}, \quad b_{\text {min }} \leq b \leq b_{m}} \\
& {\left[g^{q 4}+g^{q 4} *\left(\frac{b_{j}^{t c}-b_{m}^{t c}}{b_{\text {max }}^{t c}-b_{m}^{t c}}\right)^{\delta}\right]_{b 4}^{w_{b 4}^{t c}}, b_{m} \leq b \leq b_{\text {max }}} \\
& 1 \quad b>b_{\text {max }}
\end{aligned}
$$

where

$b_{i}^{t c}$ is the bandwidth allocated to a traffic class in the third quadrant; $b_{\text {min }}^{t c}$ is the minimum bandwidth allocation to a traffic class in the third quadrant; $b_{\text {max }}^{t c}$ is allowable maximum bandwidth allocation to a traffic class in the third quadrant; $w_{b 3}^{t c}$ is the preference weight for a bandwidth of a traffic class in the third quadrant; $w_{b 4}^{t c}$ is the preference weight for a bandwidth of a traffic class in the fourth quadrant; $g^{q 3}=$ $g^{q 4}=0.5$ are the centers of the utility , $\delta$ is the user bandwidth sensitivity index. 
Table 1. The proposed Utility Model for Multi-CriteriaMulti-Quadrant Approach

\begin{tabular}{|c|c|c|c|}
\hline \multirow[t]{2}{*}{ The Utility is 1 , if } & & & \multirow{2}{*}{$\begin{array}{l}\text { The Utility is } \\
1 \text {, if } \\
\quad b \geq b_{\max }\end{array}$} \\
\hline & $\begin{array}{l}\text { The utility } \\
\text { decreases with an } \\
\text { increase in price. }\end{array}$ & $\begin{array}{l}\text { The utility } \\
\text { increases with the } \\
\text { increase in tbe } \\
\text { bandwidth } \\
\text { allocated. }\end{array}$ & \\
\hline $\begin{array}{l}\text { The utility is } \\
\text { greater than } 0.5 \\
\text { and less than } 1\end{array}$ & First Quadrant & Fourth Quadrant & \\
\hline \multicolumn{4}{|c|}{ The Center of the Utility, The Center of the Criterion } \\
\hline $\begin{array}{l}\text { The Utility is less } \\
\text { than } 0.5 \text { and } \\
\text { greater than } 0\end{array}$ & Third Quadrant & Second Quadrant & \\
\hline $\begin{array}{l}\text { The Utility is } 0 \\
\qquad b \leq b_{\min }\end{array}$ & $\begin{array}{l}\text { The utility } \\
\text { increases with the } \\
\text { increase in the } \\
\text { bandwidth } \\
\text { allocated. }\end{array}$ & $\begin{array}{l}\text { The utility } \\
\text { decreases with an } \\
\text { increase in price. }\end{array}$ & $\begin{array}{l}\text { The Utility is } 0 \\
p \geq p_{\text {max }}\end{array}$ \\
\hline
\end{tabular}

\section{MULTI-CRITERIA-MULTI- QUADRANT APPROACH (MCMQ APPROACH)}

In this scheme, the model assumed that the blocking probability is constant, the resources are adequate and the Network is not in a congestion state. This model only provides either a positive or a negative incentive to the user to utilize the resources efficiently. The effect of partitioning the resources according to the users demand is studied and appropriate mathematical formulations are proposed.

The two criteria used in this model are price and bandwidth. The so called quadrant approach can be seen to be divided in to four quadrants. Price is a downward criterion in the $1^{\text {st }}$ and $4^{\text {th }}$ quadrant. Its utility decreases in function of its value. The bandwidth constitutes an upward criterion in the $2^{\text {nd }}$ and $3^{\text {rd }}$ quadrants. Its utility increases in function of its value. The center of utility could be a value between 0 and 1 and it is a point where a user is minimally satisfied.

\subsection{Proposed Acceptance Probability for Static Congestion Control}

Given a network selection criteria vector $\boldsymbol{x}$ and the associated preference vector $\boldsymbol{w}$, a suitable multi-criteria acceptance probability is formulated from [7].

$$
U(x)=\prod_{i=1}^{n}\left(U_{i}\left(x_{i}\right)\right)^{w_{i}}
$$

as

$$
\begin{array}{r}
A\left(u_{i}, p_{i}\right)=\left[\left(1-g^{q 1} *\left(\frac{p_{i}^{t c}-p_{\min }^{t c}}{p_{\max }^{t c}-p_{\min }^{t c}}\right)^{\alpha}\right)\right]^{w_{p 1}^{t c}} \\
*\left[g^{q 2} *\left(\frac{b_{i}^{t c}-b_{\min }^{t c}}{b_{\max }^{t c}-b_{\min }^{t c}}\right)^{\delta}\right]^{w_{b 2}^{t c}}
\end{array}
$$

The sum of all the preference weights should be 1

$$
w_{p 1}^{t c}+w_{b 2}^{t c}=1
$$

$w_{p 1}^{t c}$ is the is the preference weight for bandwidth price; $w_{b 2}^{t c}$ is the preference weight for bandwidth; $\alpha$ and $\delta$ are the different user price and bandwidth sensitivity indices.

If the user is assumed to be running a streaming application, then throughput is a more significant criterion than price (the user sets higher preference weight for the throughput criterion). For other users that send Best Effort and Non-real time polling services traffic, they could give higher preference weight for the price criterion.

\subsection{Total Revenue Generated from the Two Criteria and Four Quadrant Approach}

In radio resource management, the network operator's decision metric (i.e., payoff) is mostly the revenue. The revenue, calculated over $\mathrm{N}$ users requesting a connection to the network, is: $R=\sum_{i=1}^{N} p_{i} A\left(u_{i}, p_{i}\right)$, where $p_{i}$ is the price that user $i$ has to pay for the operator's connectivity service. Its expectation, also called potential revenue.

Then from equations(10) and (13), the total revenue generated is computed as:

$$
\begin{aligned}
& \boldsymbol{R}=\sum_{i=1}^{N_{t c}} \boldsymbol{p}_{i}^{t c} *[\left.\left(1-g^{q 1} *\left(\frac{p_{i}^{t c}-p_{\text {min }}^{t c}}{p_{\text {max }}^{t c}-p_{\text {min }}^{t c}}\right)^{\alpha}\right)\right]^{w_{p 1}^{t c}} \\
& *\left[g^{q 2} *\left(\frac{b_{i}^{t c}-b_{\text {min }}^{t c}}{b_{\text {max }}^{t c}-b_{\text {min }}^{t c}}\right)^{\delta}\right]^{w_{b 2}^{t c}}
\end{aligned}
$$

\subsection{Total Allocated Resources from the Two Criteria and Four Quadrant Approach}

In [6], the formula of the total allocated resources is given by

$$
B=\sum_{i=1}^{N} b_{i} A\left(u_{i}, p_{i}\right)
$$

and by substituting the formula of the acceptance probability model (13) in the above equation, the total allocated resources could be expressed as :

$$
\begin{aligned}
\boldsymbol{B}=\sum_{i=1}^{N_{t c}} \boldsymbol{b}_{i}^{t c} *[( & \left.\left.1-g^{q 1} *\left(\frac{p_{i}^{t c}-p_{\text {min }}^{t c}}{p_{\text {max }}^{t c}-p_{\text {min }}^{t c}}\right)^{\alpha}\right)\right]_{t_{p 1}^{t c}}^{w_{p}^{t c}} \\
* & {\left[g^{q 2} *\left(\frac{b_{j}^{t c}-b_{\text {min }}^{t c}}{b_{\text {max }}^{t c}-b_{\text {min }}^{t c}}\right)^{\delta}\right]^{w_{b 2}} }
\end{aligned}
$$


where $\boldsymbol{p}_{i}^{t c}$ Price paid by admitted traffic class; $\boldsymbol{p}_{\text {min }}^{t c}$ Minimum Price quoted to traffic class; $\boldsymbol{p}_{\max }^{t c}$ Maximum Price quoted to a traffic class; $b_{i}^{t c}$ Bandwidth allocated to admitted a traffic class; $b_{\min }^{t c}$ Minimum bandwidth allocation to a traffic class; $b_{\max }^{t c}$ Allowable Maximum bandwidth allocation to a traffic class; $N_{t c}$ Total number of admitted traffic class; $w_{p 1}^{t c}$ Preference weight for a price of traffic class in the first quadrant; $w_{b 2}^{t c}$ Preference weight for a bandwidth of traffic class in the third quadrant; $g^{q 1}=g^{q 2}=1$. (normalizing constants)

\section{THE BEHAVIOR OF THE PROPOSED AND VUONG MODELS}

It should be observed at this point that most common utility functions will be simply increasing, possibly having a sigmoid shape as shown in Figure 1. However, a utility function also might show a step pattern. This may happen if a user would like to use services with different quality-of-service requirements (in this case, QoS factor is the bandwidth). Note that the two cases correspond somehow to the notion of QoS paybacks in the marketing perspective of pricing. Still, the utility functions may often be treated as strictly concave within the [0 100] range, assuming that $\$ 0$ and $0 \mathrm{Mbps}$ are correctly chosen to be the minimum BW price and bandwidth respectively. The bandwidth $60 \mathrm{Mbps}$ and the BW price $\$ 40$ at the center of utility $(0.5)$, should be guaranteed in the SLA made between a user and a network operator. Moreover, a user should pay an incremental fee for bandwidth utilization within $[0,60 \mathrm{Mbps}]$ range and make extra payments for extra utilization within the range [60Mbps, 100Mbps]. Minimum and maximum bandwidth ranges (bmin, bmax) control the maximum and the minimum bandwidth any user may request at any given time.

Figure 2 shows how the NSP must decrease the BW price per unit of resource if the total amount of resources increases with the same user base. This decrease in unit BW price is necessary if resource utilization is to be maximized which also serves as an incentive for the users. The net utility of users increases with more resources. For initial increase in resource the utility increased very quickly from 0 but the utility slowly saturates indicating that more resources have limited value beyond a certain point $(0.5)$, i.e., the users will not find ways to utilize abundant resources.

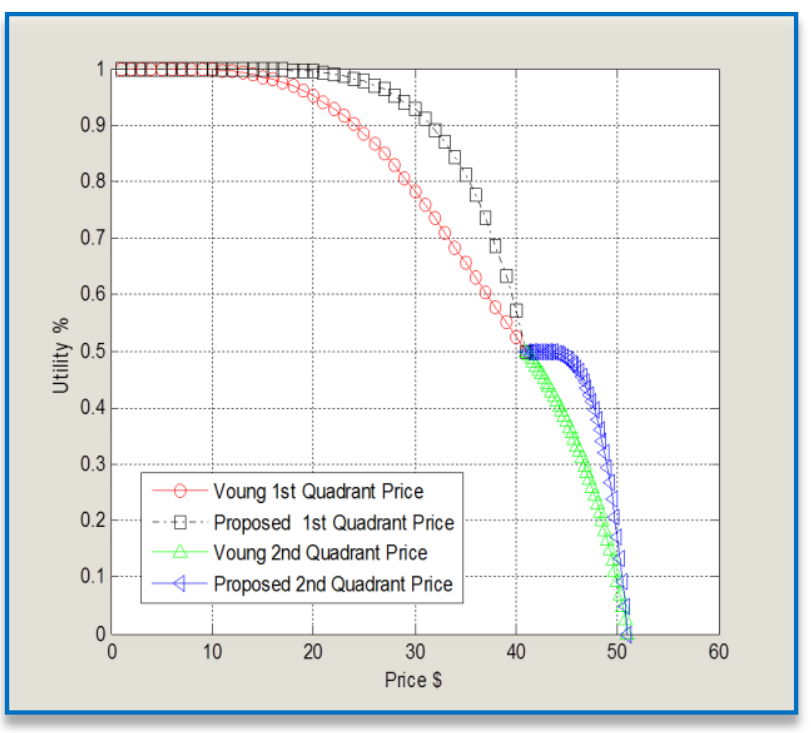

Figure 1: Utility vs. Bandwidth (BW) Price

\section{RESOURCE EFFICIENCY INDEX}

To determine jointly the optimal CAC strategy at the best pricing scheme, the network provider's point of view, mainly in terms of resource efficiency index is introduced. This is defined as the ratio of the potential revenue and the total resource allocated. Revenue is not only a function of BW price, but depends also on the users' degree of satisfaction, i.e. perceived QoS as depicted in microeconomics by utility functions, and their willingness to pay. This is called the acceptance probability. The pricing strategies shall focus on flat and service-based pricing. In the former, a user pays a fixed amount of money per time unit, regardless of the changing performance he might experience throughout the duration of his call.

The following metric in [7] is used for comparative and performance analysis of the proposed and the existing model.

$$
\boldsymbol{E}=\frac{\boldsymbol{R}}{\boldsymbol{B}}
$$

$\mathbf{E}=$ Resource Efficiency Index, $\boldsymbol{R}=$ Total revenue generated from the admitted users and $\mathbf{B}=$ Total Allocated Resources.

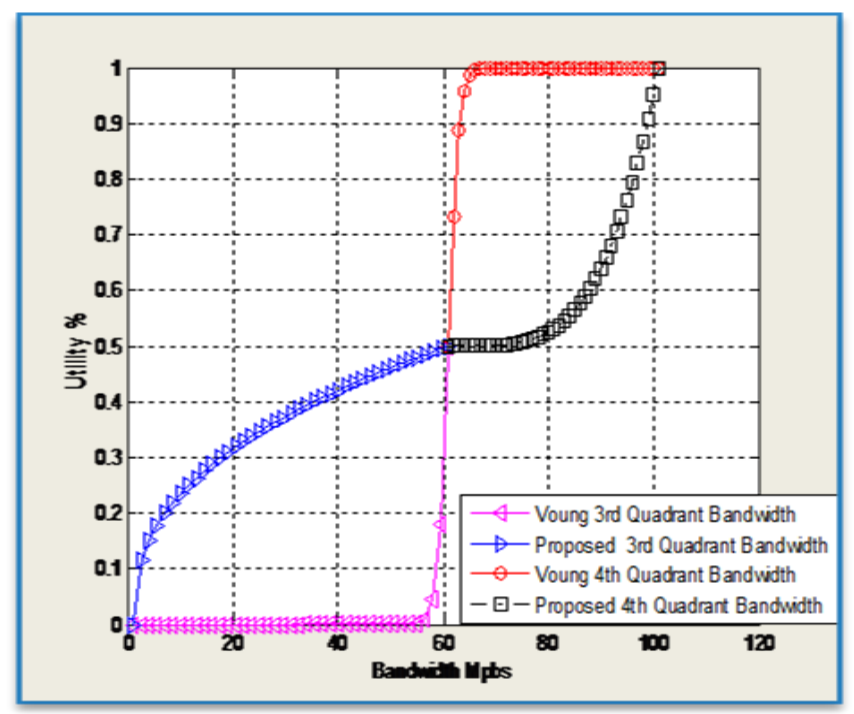

Figure 2: Utility vs. Bandwidth (BW)

\section{PERFORMANCE ANALYSIS}

In this section, the proposed model's acceptance probability for the network operator's radio resource allocation is investigated. The modified sigmoid function in Vuong Q.N (2008) is compared with the proposed model. A radio resource allocation scenario (one access network operator and $N$ users) is considered. If the user is assumed to be running a streaming application, then throughput is a more significant criterion than price (the user sets higher preference weight for the throughput criterion). For other users that send best effort and non-real time polling services traffic, they could give higher preference weight to the price criterion. This scenario is taken in this simulation. Bandwidth weight index $=0.3$, price weight index $=0.7$ and Center of utility $=0.5$ are assigned. Other values of the center of utility could be chosen depending on the application. Traditional data applications such as web browsing or email are perfectly elastic since they are assumed to tolerate variations in delay and to be able to use even the minimal amount of bandwidth. On the other hand the utility for a criterion like RSS cannot always be concave, especially for small values. A minimum level of RSS is required to establish and maintain the connectivity. 
Different users will assign different values to a given application and it's QoS [7]. Price range is $\$ 0-60$ and the Bandwidth at the center of utility is $60 \mathrm{Mbps}$.

Figure 3 depicted the performance of the two models using Bandwidth (BW) price-weight indices 0.3 and bandwidthweight index 0.7 for the Vuong and the proposed. The proposed model out performed Vuong model in terms of Cumulative Resource Efficiency (CRE) index by $6 \%$ when the realized Cumulative Revenue reaches $\$ 800$. The maximum Cumulative Revenue of $\$ 1600$ at a CRE of 0.618 , is realized by the proposed model against the maximum Cumulative Revenue of $\$ 820$ realized by the Vuong model at a CRE of $59 \%$. This implies that the proposed model can generate more revenue than the Vuong model at almost all the CREs.

Table 2: The Simulation Parameters for Vuong, and the Proposed Models

\begin{tabular}{|l|l|}
\hline Parameters & Settings \\
\hline Bandwidth range & $0-100 \mathrm{Mbps}$ \\
Bandwidth weight index & 0.3 \\
Price weight index & 0.7 \\
Center of utility & 0.5 \\
Price range & $\$ 0-60$ \\
Price at the center of utility & $\$ 40$ \\
Bandwidth at the center of utility & $60 \mathrm{Mbps}$ \\
Vuong and proposed utility & \\
Parameters & 2 \\
$\delta$ & 3 \\
$\gamma$ & \\
\hline
\end{tabular}

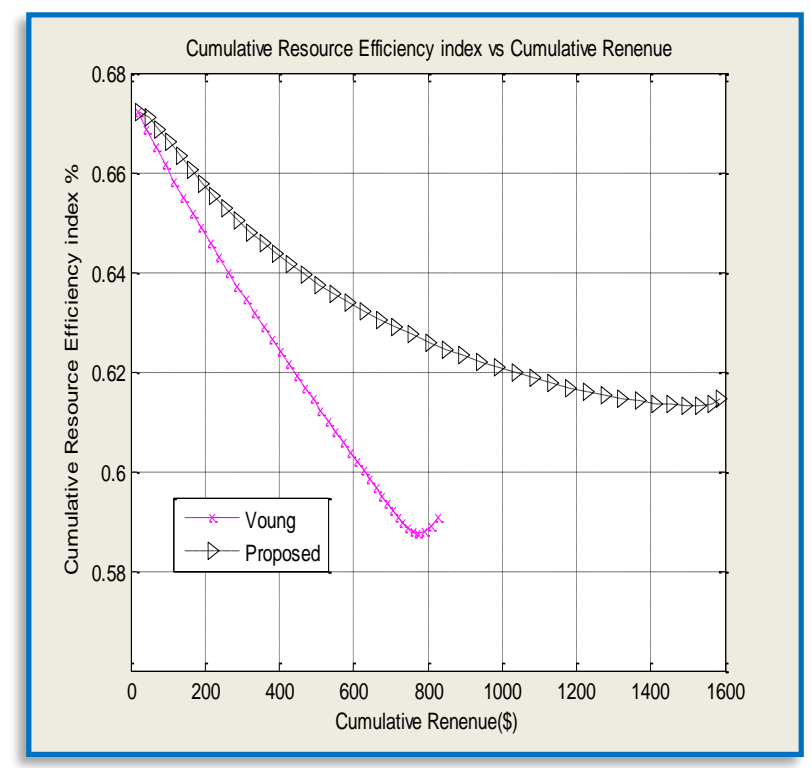

Figure 3: Cumulative Resource Efficiency vs. Cumulative Revenue
Figure 4 shows that the Cumulative Resource Efficiency (CRE) index in the proposed pricing and utility scheme has outperformed the Vuong model by $6 \%$ at Cumulative Resource Allocated (CRA) of $1450 \mathrm{Mbps}$. The maximum CRE index achieved by both models is $67 \%$ and the minimum CRE indices achieved by Vuong model and the proposed model are $59 \%$ and $61.8 \%$ respectively. The minimum Cumulative Resource Allocated (CRA) in Mbps by Vuong model and the proposed model are 2530Mbps and 1450Mbps respectively, both calculated at the minimum CRE index. This implies that the proposed model allocates $1080 \mathrm{Mbps}$ more than the Vuong model.

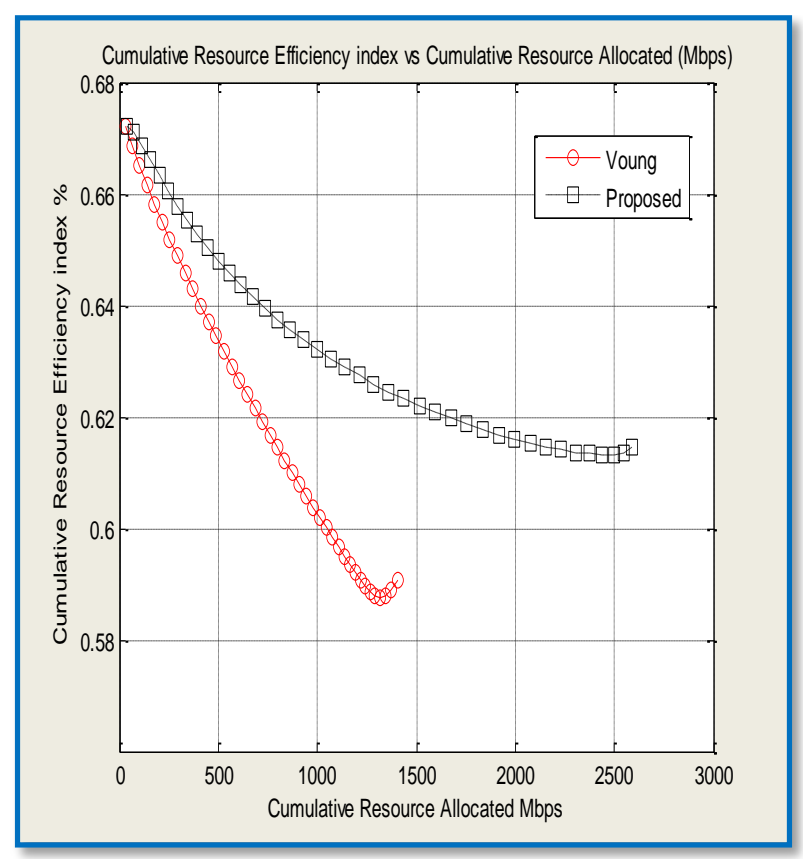

Figure 4: Cumulative Resource Efficiency vs. Cumulative Resource Allocated

Figure 5 depicted the performance of the two models using Bandwidth (BW) price-weight indices 0.3 and bandwidthweight index 0.7 for both the Vuong and the proposed. The Cumulative Resource Efficiency (CRE) index is plotted against Bandwidth (BW) price. In the incentive region, the proposed model out performed Vuong model in terms of Cumulative Resource Efficiency (CRE) index by $4.7 \%$ at the $\mathrm{BW}$ price of $\$ 41$. In the congestion region the proposed model out performed Vuong model in terms of Cumulative Resource Efficiency (CRE) index by $4.7 \%$ at the BW price of $\$ 51$. This implies that the higher the price, the lower the CRE in both models.

Figure 6 depicted the performance of the two models using Bandwidth (BW) price-weight indices 0.3 and bandwidthweight index 0.7 for both the Vuong and the proposed. The Cumulative Resource Efficiency (CRE) index is plotted against Bandwidth (BW). In the incentive region, the proposed model out performed Vuong model in terms of Cumulative Resource Efficiency (CRE) index by $4.7 \%$ at the $\mathrm{BW}$ of $61 \mathrm{Mbps}$. In the congestion region the proposed model out performed Vuong model in terms of Cumulative Resource Efficiency (CRE) index by $4.7 \%$ at the BW of $101 \mathrm{Mbps}$. This implies that the higher the resources allocated, the lower the CRE in both models. 


\section{CONCLUSIONS}

Simulations have been conducted for different values of BW prices in $\$$ and the resources allocated in Mbps. For each simulation, the resource efficiency indices in the two approaches are computed. By using the proposed utility-based acceptance probability metric, the network operator can improve resource efficiency between $6 \%$ and $7 \%$ compared to the use of the Vuong model. The results confirm that our proposed utility model also serves operators better in terms of revenue generated. In the future work, the two traffic classes like Variable Bit Rate (VBR) and Constant Bit Rate (CBR) should be considered in the analysis.

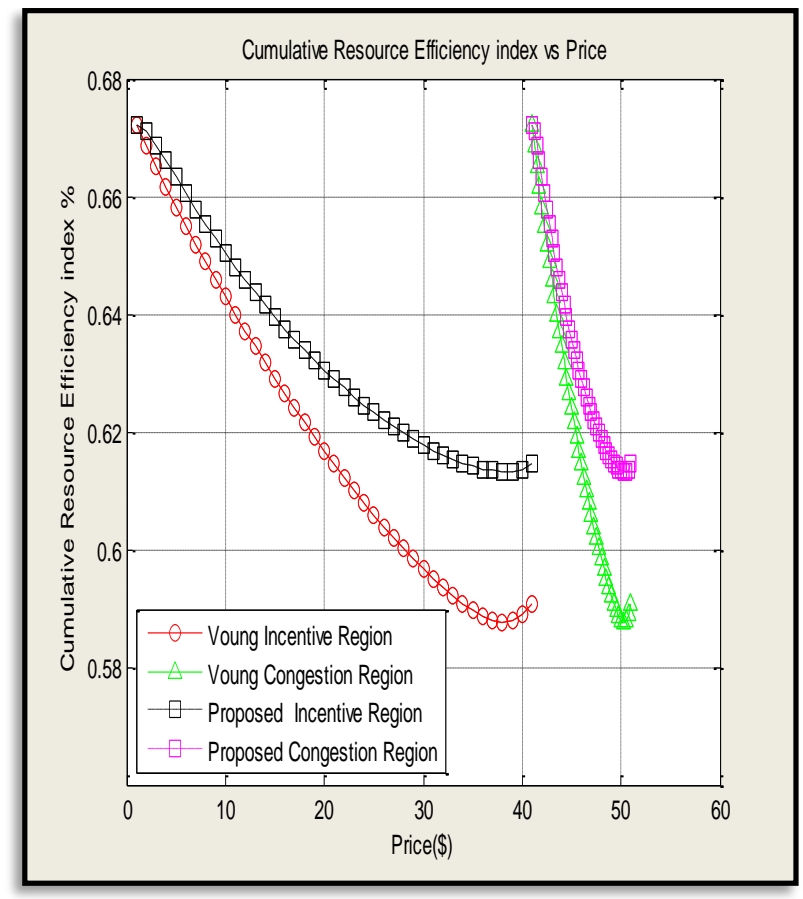

Figure 5: Cumulative Resource Efficiency vs. BW Price

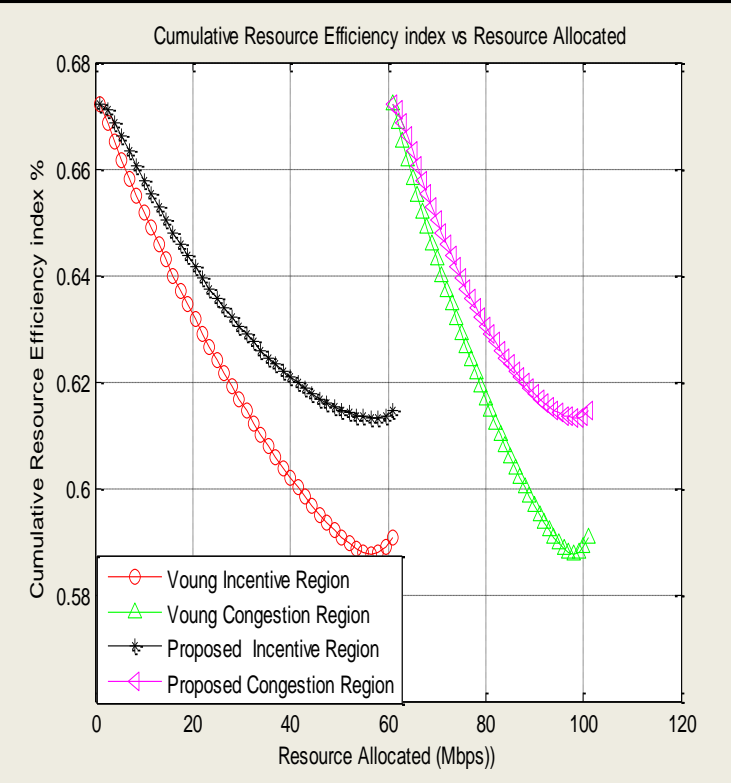

Figure 6: Cumulative Resource Efficiency vs. Resource Allocated

\section{ACKNOWLEDGEMENT}

We wish to thank the management of Nigerian Communications Commission NCC and Electrical Department of Bayero University Kano for their financial support. We also extend our gratitude to Dr. Dahiru Sani Shuaibu and Dr. Yakubu Suleiman Baguda for their intellectual guidance and support.

\section{REFERENCES}

[1] Yaipairoj, S. (2006). Enhancing Performance of Mobile Services through Pricing Mechanisms. A Thesis Submitted in partial fulfillment of the requirements of Stevens Institute of Technology for the Degree of Doctor of Philosophy.

[2] Fishburn, P.C. and Odlyzko, A.M. (1998). Dynamic Behavior of Differential Pricing and Quality of Service options for the Internet, in IC E '98, pp. 128-139.

[3] DaSilva, L. (2000). Pricing of QoS-Enabled Networks. A Survey. IEEE Communications Surveys \& Tutorials, 2000 Vol. 3, no. 2. pp 1-8.

[4] Andrews J. G., Ghosh A. and Muhammad R. (2007). Fundamentals of WiMAX. Understanding Broadband Wireless Networking. NJ, USA. Prentice Hall PTR, 2007. Andrews J.

[5] Badia L., Lindstrom M., Zander J. and Zorzi M. (2004a). An Economic Model for the Radio Resource Management in Multimedia Wireless Systems, Computer Communications, Vol.27, pp.1056-1064

[6] Badia L., Lindstrom J. and Zorzi M (2004b). On Utilitybased Radio Resource Management with and without Service Guarantees, Proceedings of the 7th ACM International Symposium on Modeling, Analysis and Simulation of Wireless and Mobile Systems Venice, Italy, pp. 244-251.

[7] Vuong Q.N (2008). Mobility Management in 4G Wireless Heterogeneous Networks. A Thesis Submitted in partial fulfillment of the requirements of Universite D'evry Val-D'essonne for the Degree of Doctor of Philosophy. 